\title{
Psammetich II. in Palästina und in Elephantine.
}

Von Yrivatdozent Ijic. Albrecht Alt in Greifswald.

Die politischen Vorgänge, die sich im letzten Jahrzehnt vor der Zerstörung Jerusalems durch die Chaldäer auf dem Boden von Palästina abgespielt haben, sind uns nur sehr unvollkommen bekannt. Das biblische Buch der Könige redet mit kurzen Worten von der Erhebung Sedekias, des letzten Königs von Juda, auf den Thron der Davididen und erzählt ausführlich von seinem und Jerusalems Untergang; die dazwischenliegenden Jahre hingegen übergeht es mit ein paar schematischen Angaben über Ședekias Alter und Herkunft, über Dauer und Art seiner Regierung ${ }^{\mathrm{x}}$. Die klaffende Lücke, die bei dieser literarischen Darstellung bestehen bleibt, - wird nun leider auch durch keine inschriftliche Nachricht aus den Nachbargebieten geschlossen: Nebukadnezars des Chaldäers umfangreiche Inschriften schweigen sich nach echt babylonischem Brauch über die politischen Ereignisse seiner Zeit völlig aus, und ebensowenig findet sich in den spärlichen ägyptischen Denkmälern aus jenem Jahrzehnt irgendwelche Bemerkung, die auf palästinische Dinge bezug hätte. Selbst HERODOT, der sonst so viele wertvolle Nachrichten über die Geschichte des sechsten Jahrhunderts v. Chr. bewahrt hat, läßt unshier im Stich. 'Lediglich den Prophetenschriften Jeremias und - in beschränkterem Maße.- Hesekiels verdanken wir einigen Einblick in das bewegte politische Wechselspiel jener Zeit ${ }^{2}$.

Thre Angaben scheinen nun eine unerwartete Ergänzung und Beleuchtung $z u$ erhalten durch eine an sich unscheinbare Notiz, die sich in einer kürzlich bekannt gewordenen ägyptischen Urkunde findet3. Das Schriftstück, um das es sich handelt, bildet seinem äußeren Umfang wie seinem inneren Gehalt nach den wertvollsten Bestandteil einer Gruppe von neun altdemotischen Papyrusurkunden, die in den antiken Ruinen bei der mittelägyptischen Ortschaft el-Hîbe ${ }^{4}$ von Einheimischen gefunden

\footnotetext{
$\times 2 \mathrm{Kön} 24 \mathrm{I}_{7}-2025 \mathrm{xf}$. Vgl. den noch weiter verkürzten Bericht $2 \mathrm{Chr} 36 \mathrm{Irff}$.

2 Besonders Jer $27-29$ Hes 12 I7.

3 Den ersten Hinweis auf sie verdanke ich einer gütigen Mitteilung von Herrn Geheimrat Professor Dr. ERMaN in Berlin.

4 Die moderne Ortschaft liegt auf einer Nilinsel, die antiken Kiuinen auf dem rechten Ufer, der Ortschaft Feschn gegenüber. Vgl. zur Ortslage Annales du Serv. des
} 
und im Winter 1898/99 durch Lord Crawford käuflich erworben und nach Europa gebracht worden sind. Mittlerweile sind sie in die große John Rylands Library in Manchester übergegangen und im vorigen Jahre mit mehreren Gruppen jüngerer demotischer Papyri zusammen von F. Ll. GRIFFITH mustergültig veröffentlicht worden ${ }^{x}$. Die Urkunden von el-Hibe oder Teuzoi - so umschreibt GrIFFITH den in ihnen gebrauchten altägyptischen Namen des Ortes - gehören auch inhaltlich zusammen: sie beziehen sich ohne Ausnahme auf Angelegenheiten einer und derselben Familie, die während einer Reihe von Generationen in Teuzoi wohnte und zeitweilig priesterliche Ämter an dem dortigen Amontempel ${ }^{2}$ bekleidete. Offenbar entstammen die Urkunden dem Hausarchiv jenes Beamten- und Priestergeschlechts - ein Tatbestand, wie er ja z. B. auch bei den von SAYCE und COWLEY veröffentlichten jüdisch-aramäischen Urkunden aus Elephantine vorliegt.

Während nun die älteren acht unter jenen Papyri 3 einfache Geschäftsurkunden mancherlei Inhalts sind, stellt sich der jüngste und längste von ihnen - derjenige, auf den es uns hier ankommt - als eine umfangreiche Beschwerdeschrift dar, die ein Abkömmling des genannten Geschlechts, Peteêse mit Namen, unter der Regierung Darius' I., anscheinend bald nach dessen neuntem Jahre (512 v. Chr.), an die persische Oberbehörde 4 in Memphis eingereicht hat. $\mathrm{Zu}$ ihrer Abfassung sah sich Peteêse, wie er im Eingang berichtet ${ }^{5}$, durch schwere Mißhandlungen veranlabt, die er von seiten der Priesterschaft von Teuzoi erlitten hatte und für die ihm bei der persischen Regierung trotz aller Bemühungen keine hinreichende Sühne zuteil geworden war. Der Aufforderung des persischen Beamten folgend, sucht er nun seine Rechtsansprüche historisch zu begründen, indem er die wichtigsten Vorfälle aus der Geschichte der Beziehungen seiner Familie zu dem Tempel und den Priestern von Teuzoi mit großer Ausführlichkeit schriftlich darlegt ${ }^{6}$; zeitlich weit auseinander-

Antiqu. II (1901) 84ff. 154ff.; Egypt Explor. Fund Archaeol. Report 1901/02 4 f. 1903/03 Iff.; Grenfell and Hunt The Hibeh Papyri I (1906) Iff.; Bardeker Ägypten 6 I 98.

I Catalogue of the Demotic Papyri in the John Rylands Library Manchester. Manchester and London 1909; 3 voll. (im folgenden einfach mit „Griffith“ zitiert).

2 Es ist derselbe von den Königen der XXII. Dynastie begründete Tempel, dessen beute fast ganz zerstörte Inschriften wahrscheinlich ein Duplikat zu der berühmten Scheschonkliste des Amontempels von Karnak enthielten; vgl. Ann. du Serv. II 155 f.

$3 Z_{\text {wei }}$ entstammen der Zeit Psammetichs I., sechs den Jahren Amasis'.

4 Die Bezeichnung des obersten Beamten, mit dem es Peteêse zu tun hat, ist nicht ganz durchsichtig; wahrscheinlich ist der persische Satrap gemeint. Vgl. GRIFFITH III 61 .

5 IX $I_{2}-5 x_{3}$ (Zählung nach Griffith). 6 IX $5 x_{3}-2 I_{9}$. 
liegende Ereignisse aus den Regierungsjahren Psammetichs I., Psammetichs II. und Amasis' bringt er dabei ohne besondere Verbindung in cine chronologische Reihenfolge. Zum Schluß fügt er seiner Beschwerde noch die Abschriften mehrerer alter Urkunden an, deren Wortlaut Peteêses eigenen Bericht bestätigen soll $\mathrm{x}$.

Uns geht vor allem der mittlere Abschnitt jenes geschichtlichen Rückblicks an, der Abschnitt über Ereignisse aus der Zeit Psammetichs II $^{2}$. Peteêse erzählt hier, im vierten Jahre des Pharao Psammetich Neferebrê (d. i. Psammetich II.) seien zu allen grolien Tempeln Ägyptens Boten gesandt worden mit der Meldung: „Der Pharao zieht nach dem Lande Chor" und mit der Weisung, daß von den Tempeln Priester mit den Abzeichen(?) ihrer Götter zur Begleitung des Königs entsandt werden sollten. Von den Priestern von Teuzoi sei daraufhin des Erzählers gleichnamiger Großvater, der Amonprophet Peteêse, abgeordnet worden, und dieser habe auch wirklich den Zug nach Chor mitgemacht. Während seiner Abwesenheit sei sein Amt von den Priestern einem anderen übertragen worden, und er habe nach seiner Rückkehr nicht wieder in den Genuß sèiner Rechte gelangen können, da der Pharao bald darnach krank geworden und gestorben sei.

Ist der hiermit verkürzt wiedergegebene Bericht Peteêses glaubwürdig, so lernen wir durch ihn ein bisher ganz unbekanntes Ereignis der politischen Geschichte kennen: einen Zug Psammetichs II. in seinem vierten Jahre - d. i. 590 v. Chr. ${ }^{3}$ - nach Chor - d. i. Palästina 4. Welcher Art dieser Zug war, wird von Peteêse. mit keinem Worte angegeben; aber wir dürfen uns über dieses Schweigen durchaus nicht wundern. Dem Verfasser einer Beschwerdeschrift kommt es natürlich nicht-im geringsten auf Mitteilungen aus dem Gebiet der politischen Geschichte an, nur die persönlichen Erlebnisse seiner Ahnen will er schildern; alles andere wäre für ihn und seine $Z$ wecke nur störendes Beiwerk. $\mathrm{Da} B$ nach der Lage der Dinge ein Zug Psammetichs II. nach Palästina politischer und militärischer Natur gewesen sein muß, ist ohnehin klar.

Aber dürfen wir Peteêses Angaben überhaupt Glauben schenken?

I IX $2 \times$ ro-25 9.

2 IX 146-I6I; eine kurze Erwähnung derselben Vorgānge findet sich schon in der Einleitung $3 \mathrm{x}_{5}-4 \mathrm{x}$.

3 Zur Chronologie vgl. besonders BREASTED Ancient Recoräs of Egypt IV SS $9 \mathrm{~S}_{4} \mathrm{f}$. Psammetich II. regierte von 594 oder 593 bis 588 .

4 Über die Bedeutung des Namens Chor (in den Inschriften des Neuen Reiches syllabisch geschrieben h3 - rw) siehe W. M. MüLLER Asien und Europa 148 ff.; GRIFFITH III 96318. 
Was seine Berichterstattung über Ereignisse der Zeit Psammetichs I. betrifft, so hat GRIFFITH gegen deren geschichtliche Treue berechtigte Bedenken geltend gemacht ${ }^{x}$; und so ist natürlich auch bei dem für uns in Frage stehenden Abschnitt die Möglichkeit nicht schlechthin von der Hand zu weisen, daß dem Verfasser Irrtümer und Schreibfehler unterlaufen sind oder daß er absichtlich eine falsche Darstellung gegeben hat. Allein bei ruhiger Erwägung des Sachverhalts wird man dieser logischen Möglichkeit keine allzugroße Wahrscheinlichkeit beimessen können. Gegen sie spricht schon die ganz nebensächliche und sozusagen unbetonte Art, in der jenes Psammetichfeldzugs Erwähnung geschieht; der Verfasser kann ihn nicht umgehen, aber er schenkt ihm, wie wir vorhin sahen, auch nicht mehr Beachtung, als bei seinen besonderen $Z$ wecken erforderlich ist. Nach Tendenz klingen seine Worte jedenfalls gar nicht. Sodann besteht zwischen den von GRIFFITH mit Recht beanstandeten Erzählungen aus der Zeit Psammetichs I, und unserm Abschnitt doch in mehr als einer Beziehung ein beachtenswerter Unterschied. Dort handelt es sich um Fragen der Organisation, Titulatur und zeitlichen Reihenfolge gewisser Beamtenkategorien, also um Dinge, die sich nach I 50 Jahren nicht mehr so leicht kontrollieren ließen, hier dagegen um ein einschneidendes Ereignis der großen Politik, das einfach zu erfinden der Verfasser einer Eingabe nicht wagen durfte, ohne sich der Gefahr sofortiger Entlarvung durch die Beamten der obersten Landesbehörde auszusetzen. Auch stand Peteêse zeitlich den Ereignissen des Jahres 590 nicht allzuferne; als er bald nach $5^{\mathrm{I} 2}$ seine Beschwerde schrieb, war er ein alter $\mathrm{Mann}^{2}$, und wenn wir auch sein Geburtsjahr nicht genau kennen, so zeigt doch ein auf seinen Namen ausgestellter Kontrakt aus dem achten Jahre des Amasis (d. i. 562 v. Chr.3), daß er kaum sehr viel später als ein Jahrzehnt nach jenem Feldzug des Psammetich II. geboren sein wird. Was sein Grobvater damals erlitt, war zudem für die Geschichte der ganzen Familie von so nachhaltiger Wirkung, daß wir wohl zu der Annahme berechtigt sind, die Erinnerung daran habe sich auf Sohn und Enkel vererbt4.

Das gute Zutrauen zu Peteêses Bericht, das sich aus den eben angestellten Erwägungen ergibt, wird kaum erschüttert durch den Hinweis auf die Tatsache, daf in dem uns anderweit bekannten geschichtlichen Überlieferungsstoff keine direkte Bestätigung jener Angabe zu finden ist;

I GrIfFith III 72 f., $106 \mathrm{f} . \quad 2$ IX 1 ז $4 . \quad 3$ Pap. VIII.

4 Eine sorgfältige Abwägung der Wahrscheinlichkeitsgründe gibt GrIFFltir III $92 \mathrm{f}$. 
cine solche fordern hielic von dem dürftigen Material, das uns zu Gebote steht, zuvicl verlangen. Ja man wird im Gegenteil als ein veiteres Moment zu den aufgezeigten Stützen der Glaubwürdigkeit die Beobachtung hinzufügen dürfen, dali sich Peteises Angabe seirr wohl in den Rahmen dessen fügt, was wir sonst über die politischen Bewegungen jener Zeit wissen. Ein Feldzug Psammetichs II. nach Palästina bedeutet nichts anderes als cin neues Glied in der langen Reihe von Versuchen der Pharaonen, dic altererbten Ansprüche Ägyptens auf Südsyrien der vorderasiatischen Grolimacht gegenüber $z u$ erneuern und durchzusetzen; man denke - um nur Angehörige der XXVI., saïtischen Dynastie zu nennen - an Psammetich I. ${ }^{2}, \mathrm{Necho}^{2}$, Apries ${ }^{3}$. Tritt nun noch Psammetich II. in die Reihe, so fehlt kein Glied mehr in der Kette, und die Tatkraft, mit der die Saïten die Grundsätze ihrer asiatischen Politik durchzuführen bestrebt waren, tritt nur noch deutlicher ins Licht. Eben dieser gröljere $\mathrm{Zu}$ sammenhang der politischen Geschichte verleiht den vorhin beigebrachten Wahrscheinlichkeitsbeweisen für die Zuverlässigkeit Peteêses in dem fraglichen Punkte erst die rechte Krönung. Versuchen wir daher nun, seine Angabe, unter Voraussetzung ihrer Glaubwürdigkeit dem Rahmen der uns sonst aus jener Zeit bekannten Ereignisse einzufügen.

Die letzte entscheidende Wendung in dem Schicksal Syriens und Palästinas vor Psammetichs II. Zeit war mit der Schlacht bei Karkemisch im Jahre 605 eingetreten: die kurze Herrlichkeit von Nechos Suprematie bis an den Euphrat ging damit zu Ende, Nebukadnezar ergriff als Erbe Assurs von der ganzen Provinz Besitz. Aber politische Ruhe trat daraufhin noch lange nicht ein; weder wollte der Freiheitsdrang der syrischen Kleinstaaten, der bei dem Zusammenbruch des assyrischen Großreiches von neuem entflammt war, so schnell erlöschen, noch war Ägypten bereit, seine alten Ansprüche auf asiatisches Gebiet ohne weiteres aufzugeben. Infolgedessen zeigen uns die nächsten zwei Jahrzehnte einen Ausbruch der politischen Gärung nach dem andern: einen allgemeinen syrischen Aufstand, den Nebukadnezar um 602 niederwirft', den Abfall des Jojakim von Juda, dem im Jahre 598 oder 597 die erste Deportation nach Babylonien folgt 5 , eine Konspiration palästinischer Kleinstaaten um

$x$ Herodor II 157 . 22 Kön 2329 ff. HERodot II 159.

3 Jer 375 ff. HERODOT II $16 \mathrm{I}$.

4 Nach einem keilinschriftlichen Fragment bei WINCKLER Keilinschr. Textbuch $2{ }_{5} \mathrm{~S}$. Viclleicht gehört auch 2 Kôn $24 x$ hierher; vgl. WincKLER KAT 3278 und dagegen KitTeL Gesch. d. Hebräer II 2549 .

52 Kön 24 xo ff.

4. 10. 10. 
594, die der Prophet Jeremia bekämpft ${ }^{\mathrm{x}}$, und schließlich die Erhebung Ședekias, die zur. Zerstörung von Jerusalem 586 führt $^{2}$. Daß bei all diesen Bewegungen $\ddot{g} g y p t e n$ nicht unbeteiligt war, ist längst vermutet worden; die Analogie der Geschichte der Assyrerzeit legt eine solche Vermutung von vornherein nahe, und für das letzte der angeführten Ereignisse, für Ședekias Erhebung, haben wir in der Drohweissagung Hesekiels $^{3}$ und in dem erzählenden Bericht des Buches Jeremia 4 das unmittelbare Zeugnis einer ägyptischen Aktion. Bisher hatte man meinen können, erst Apries habe bei diesem Anlaß den Weg politischer Zurückhaltung verlassen, den sein Großvater Necho nach dem Tag von Karkemisch zu betreten genötigt wars; nun zeigt uns die Angabe Peteêses, $\mathrm{da} B$ Apries mit seinem tätigen Eingreifen in die asiatische Politik nur das Werk seines Vaters fortsetzte, daß schon Psammetich II. über die rein diplomatische Beteiligung wieder hinausgegangen war und sich persönlich in die syrischen Händel gemischt hatte.

$\mathrm{Ob}$ wir nun freilich Psammetichs Feldzug mit einem der aufgezählten Ereignisse in Palästina in unmittelbare Verbindung setzen dürfen, erscheint fraglich. Man könnte versucht.sein, in ihm eine letzte Folge jener von Jeremia bekämpften Konspiration der Kleinstaaten zu sehen; aber der zeitliche Abstand der beiden Vorgänge ist doch reichlich groß, und vielleicht hat auch jene Konspiration gar keine weiteren Folgen gehabt ${ }^{6}$. Mit größerer Wahrscheinlichkeit läßt sich Psammetichs Zug mit Ședekias Abfall von Nebukadnezar in Zusammenhang bringen, etwa in dem Sinne, $\mathrm{da} ß$ Psammetich durch seine Expedition den Anschluß - oder, ägyptisch gesprochen, die Unterwerfung - Ședekias und der übrigen Fürsten erzwang, oder auch so, daß Psammetichs Zug schon die Folge jenes Bündnisabschlusses war, den wir in Hes 12 werden und in Hes 17 vollendet sehen. Daß Nebukadnezar erst im Anfang des Jahres $587 \mathrm{zu}$ energischen Mabnahmen gegen den nach dieser Auffassung schon im Jahr $590^{\prime}$ geschaffenen Stand der Dinge schritt, darf uns nicht verwundern; auch in'dem Bericht des Königsbuches steht ja die Angabe über Ședekias

' Jer $27 \mathrm{f}$; ; die Umänderung des Datums von 27 I nach 28 ' setze ich als allgemein anerkannt voraus (vgl. besonders KUENEN, hist.-krit. Einleitung S 54).
22 Kön 2420 b 25.
3 Hes 17 is ff.
4 Jer $375 \mathrm{rr}$.
52 Kön 247.

6 Dies letztere wäre erwiesen, wenn Jer $5^{\mathrm{I}} 59$ zeitlich unmittelbar hinter Jer $27 \mathrm{f}$. gehörte und für das Verhalten auch der anderen Kleinstaaten typisch wäre - eine durchaus inögliche Annahme.

7 Nach der Analogie der asiatischen Feldzüge des neuen Reiches dürftc man auch bei Psammetich IL an den Sommer als die Zeit seiner Expedition denken; vgl. BREASTED Äg. Zeitschr. XXXVII. (1899) 129. 
Abfall von der Zeitbestimmung für den Beginn der Belagerung von Jcrusalem deutlich getrennt ${ }^{x}$, und wir wissen nicht, welche Unternehmungen Nebukadnezar in der Zwischenzeit durchzuführen hatte ${ }^{2}$.

Sind wir aber berechtigt, Psammetichs Feldzug gerade auch mit der Geschichte des jüdischen Staatswesens zu verknüpfen, so wird es sich verlohnen, noch eine andere Überlieferung aus Psammetichs Zeit in Erwägung zu ziehen. Das einzige, was HERODOT außer einer halbgriechischen Anekdote 3 über ihn zu berichten .wei $\emptyset$, ist das, daß er sechs Jahre regiert habe und daß er nach einem Feldzug gegen Äthiopien sogleich ( $\mu \varepsilon \tau \alpha u \tau i k \alpha)$ gestorben sei ${ }^{4}$. Dieser äthiopische Feldzug, der nach HERODOTs Angabe wohl im Jahre 589, also etwa ein Jahr nach der palästinischen Unternehmung stattgefunden hat, ist in den letzten Jahrzehnten oft besprochen worden; er bietet den wichtigsten Anhaltspunkt für die Datierung der griechischen und phönizischen Söldnergraffiti, die an einem der Kolosse von Abu Simbel in Nubien eingekritzelt sind5. Trotz aller Versuche, sie auf die Zeit Psammetichs I. zurückzuführen ${ }^{6}$, wird man mit WIEDEMANN 7 und anderen diese Zeugnisse einer ägyptischen Expedition nach dem Süden dem Zuge Psanmetichs II. zuschreiben müssen. Denn alles, was wir über das Verhältnis Psammetichs I. zu dem Reich der Äthiopen wissen, deutet auf die Aufrechterhaltung friedlicher Beziehungen zwischen den beiden Staaten hin, so vor allem die sog. Adoptionsstele der Nitokris, aus der hervorgeht, daß im neunten Jahre Psammetichs I. dessen Tochter Nitokris von dem "Gottesweib" des thebanischen Amon, der Schepenwepet, der Schwester Taharkas, adoptiert und damit der friedliche Übergang der Thebais, des von jenem "Gottesweib" beherrschten geistlichen Fürstentums, aus der äthiopischen Oberhoheit in den Besitz der saïtischen Dynastie gewährleistet wurde ${ }^{8}$, desgleichen auch die Reliefdarstellungen einer mit allerlei Schätzen aus Nubien heimkehrenden Flotte im Muttempel zu Karnak, bei denen man

I 2 Kön $2420 \mathrm{~b}$ neben $25 \mathrm{r}$.

2 Als Analogie sei hier nur die Geschichte der Jahre 705-701 genannt; der syrische Aufstand, der doch sicher sofort nach Sargons Tod begonnen hatte, konnte von Sanherib erst nach mehreren Jahren niedergeworfen werden.
$3 \mathrm{HER}$. II 160.
4 HER. II I6I.
5 C. I. G. 5126 (besser RöHL

I. G. A. 482). C. I. S. I III2.

6 So besọnders Hirschrerd Rhein. Museum .N. F. XLII (1887) 209 ff. Weitere Literatur bei LARFELD Griechische Epigraphik I (1907) 403.

7 Rhein. Museum N. F. XXXV (1880) $36+$ ff.

8 Textausgabe von LEGRAIN Äg. Zeitschr. XXXV (1897) I6ff; deutsche Übersetzung und Erklärung von ERMAN ebenda 24 ff. Vgl. auch BREASTED Anc. Rec. IV SS 935-958. 
viel eher an friedlichen Austausch als an Krieg und Beute zu denken hat x. Die Steleninschrift aber, in der W. M. MÜLLER ein Zeugnis für einen sonst nirgends erwähnten Feldzug Psammetichs I. gegen Äthiopien gefunden zu haben glaubte ${ }^{2}$, gehört, wie GRIFFITH erkannt hat ${ }^{3}$, in Wirklichkeit Psammetich II. an, bestätigt also nur HERODOTs Überlieferung. Schließlich aber besteht WIEDEMANNs Nachweis ${ }^{4}$ auch heute noch zu Recht, dal die Anwesenheit des Königs Psammetich in Elephantine, von der die längste unter den griechischen Inschriften von Abu Simbel redet, ihre Bestätigung in den Felseninschriften der Umgegend von Assuan findet, wenn man sie auf Psammetich II. bezieht, während Psammetich I. ebendort keine Inschrift hinterlassen hat5. Alles berechtigt uns zu der Annahme, da $B$ zur Zeit Psammetichs $I$. in den Beziehungen

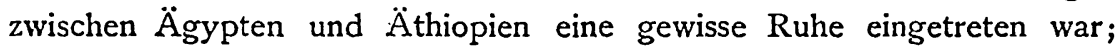
das Äthiopenreich hatte in dem halben Jahrhundert von Pi anchis erstem Vordringen nach Norden (um $720 \mathrm{v}$. Chr.) bis zu Tanutamons endgiltiger Vertreibung aus Ägypten (um 663) seine Stoßkraft erschöpft und sank immer weiter nach Süden zurück, die Dynastie der Saiten hingegen hatte zunächst in Ägypten Schwierigkeiten genug zu überwinden und suchte Anknüpfung im Norden, in Syrien und Griechenland ${ }^{6}$. Erst später kam es zu vereinzelten Zusammenstößen, unter denen unserer Kenntnis nach die Expedition Psammetichs II. die älteste ist?.

Schon WiEDEMANN aber hat gesehen, daf eine Anspielung auf diese Expedition auch in der Stelle des viel jüngeren Aristeasbriefes vorliegt, die neuerdings durch die Entdeckung der jüdisch-aramäischen Papyri aus Elephantine $z \mathrm{u}$ unerwarteten Ehren gekommen ist ${ }^{8}$. Danach sollen schon

I Veröffentlicht von BENSON and Gourlay The Temple of Mut in Asher Pl. XX bis XXII, pp. 46257370 ; vgl. GRIFFITH III 73.

a Egyptological Researches (Carnegie Instit. Public. 53) Pl. XII. XIII. p. 22 f. Mülleg hatte den Thronnamen. des Königs in Zeile 4 (Pl. XII.) nfr-lb-r' mit dem ähnlich aussehenden Thronnamen Psammetichs I. w3h-ib-r verwechselt.

3 GRIFFirH Catalogue III 320 und vorher schon Archaeol. Report 1906/07 43. Danach sind meine Bemerkungen in Israel und Agypten 89 Anm. I und ebenso diejenigen KitTels in Geschichte der Hebräer II ${ }^{2} 527$ Anm. 3 zu berichtigen.

$4 \mathrm{aal}) 366 \mathrm{f}$.

5 Vgl. die Denkmālerlisten bei W.M. Flinders Petrie History of Egypt III $325 \mathrm{ff}$. $340 \mathrm{f}$.

6 Vgl. vor allem H. SCHÄFER Die aethiop. Königsinschrift des Berl. Museums (1901) 31 ff.; Klio IV (1904) $152 \mathrm{f}$.

7 Aus späterer Zeit kommen besonders der Zug des Äthiopenkönigs Harsijôtef um 549 bis Assuan (vgl. dessen grofie Inschrift Z1. 92 ff.) und des Kambyses verunglücktes Unternehmen im Jahr 525 (Herodor III 17-25) in betracht.

8 Aristeas ed. Wendland S 13; Wiedenann aaO. 369. 


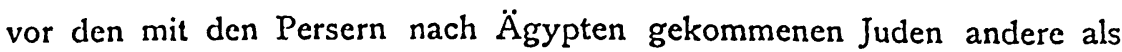
Bundesgenossen dorthin entsandt worden sein, um mit Psammetich gegen den König der Äthiopen zu kämpfen. $\mathrm{Da}$ wir nun wissen, daß in Elephantine eine jüdische Gemeinde bestand, die schon vor der Perserzeit ihr eigenes Jahweheiligtum besa $ß$ und die noch in der Perserzeit militärischen Charakter trug, so werden wir in jener Angabe des Aristeasbriefes eine gute Überlieferung sehen dürfen. Sie auf die Zeit Psammetichs I. zu deuten, wie dies STEUERNAGEL will ${ }^{x}$, haben wir aber nach den obigen Ausführungen keinen Anlaß und kein Recht; vielmehr werden wir durch Kombination des Palästinazuges Psammetichs II. und seines Äthiopenzuges zu einer neuen Anschauung geführt. Den Palästinazug vom Jahre 590 lernten wir vorhin mit großer Wahrscheinlichkeit als Anlaß oder Folge jenes „Bündnisses" zwischen Juda und Ägypten betrachten, das Hesekiel von der Ferne her bedrohte und das Ședekia und Jerusalem schließlich in den Untergang trieb; an dem Äthiopenzug vom Jahre 589 sehen wir durch Aristeas jüdische Krieger als Bundesgenossen beteiligt. Was liegt näher als die Vermutung, , daß diese jüdischen Bundesgenossen ebenso durch jenes Bündnis in den äußersten Süden von Ägypten geführt wurden wie zwei Jahre später die ägyptischen Söldner nach Pälästina $\mathrm{zu}$ ihrem vergeblichen Versuch, Jerusalem Entsatz zu bringen? In beiden Fällen hätten wir es demnach mit Erscheinungen $z u$ tun, die in dem gleichen Faktum, Psammetichs Palästinafeldzug und Ședekias Anschluß an ihn, ihre letzte Wurzel haben.

Ist diese Kombination richtig, so erklärt sich vielleicht auch die vielbesprochene Gründung eines Jahweheiligtums in Elephantine noch etwas einfacher: sie hat dann möglicherweise erst stattgefunden, nachdem in Juda. die entscheidenden Schläge gefallen waren, also um oder nach 586. Nun war für die jüdischen Söldner die Möglichkeit geschwunden, nach Ableistung ihres Dienstes in die Heimat zurückzukehren ${ }^{2}$; nun fühlten sie auch das Bedürfnis, sich in einer die Selbständigkeit ihrer Kolonie gewährleistenden Weise auf fremder Erde einzurichten. Jahwe hatte das Land verlassen ${ }^{3}$, das Diasporaleben begann. Und eben dann, wenn die Sendung jüdischer Söldner nach Ägypten der Ausfluß eines politischen

I Theolog. Studien und Kritiken 1909 I ff.

2 Die Inschrift des Neshiôr (zuletzt veröffentlicht von H. SchẌFER Klio IV [1904] i 55 ff.) aus der Zeit des Apries nennt unter den ausländischen Elementen der Besatzung von Elephantine ' $3 \mathrm{~m}$, ḩznbw und śțt „Syrer, Griechen und Asiaten"; die große Söldnerinschrift von Abu Simbel unterscheidet nur $\alpha \lambda(\lambda) \omega \gamma \lambda \omega \sigma(\sigma)$ ous und Aliviurious.

3 Hes $8 x_{2}$. 
Bündnisses war, verstehen wir auch am leichtesten, wie noch unter der Herrschaft von Pharaonen in dem fremden Einflüssen sonst so wenig zugänglichen Oberägypten ein fremder Kultus begründet werden konnte; königliche Vollmachten werden von Anfang an hinter der Gründung gestanden haben $\mathrm{x}$.

Es bedarf zum Schluß wohl keiner besonderen Hervorhebung dessen, daß die zuletzt gewagten Kombinationen stark hypothetischen Charakter an sich tragen. Den Anspruch aber werden wir wohl für sie erheben dürfen, daß sie in der Zusammenfügung sehr verschiedenartiger, gegenseitig ganz unabhängiger Quellen, auf der sie ruhen, ein Fundament besitzen, wie es andere bisher vorgetragene Meinungen nicht aufweisen können.

I Natürlich soll mit alledem die Möglìchkeit späterer Verstärkung der Judengemeinde in Elephantine durch neue Zuzüge nicht ausgeschlossen werden; nur um Feststellung des Ausgangspunktes kann es sich handeln. 